\title{
Extended Hi Rotation Curve of M31 using deep DRAO observations
}

\author{
C. Carignan ${ }^{1}$, L. Chemin ${ }^{1,2}$, and T. Foster ${ }^{3}$ \\ ${ }^{1}$ Dépt. de Physique, Université de Montréal, C.P. 6128, succ. centre-ville, Montréal (Qc), \\ Canada, H3C3J7; e-mail: [chemin,carignan]@astro.umontreal.ca \\ ${ }^{2}$ GÉPI, Observatoire de Paris, 5, pl. Janssen, 92195, Meudon, France \\ ${ }^{3}$ Dept. of Physics \& Astronomy, Brandon University, Brandon, MB, Canada, R7A 6A9; \\ e-mail: fostert@BrandonU.CA
}

\begin{abstract}
Carignan et al. (C06, 2006, ApJ, 641, L109) recently presented an extended HI rotation curve (RC) of M31, using single dish observations from the $100 \mathrm{~m}$ Effelsberg and Green Bank telescopes. These data were obtained along the semi-major axis of the approaching half of the M31 Hi disk and showed a flat RC at large radius, which extends up to $\sim 35 \mathrm{kpc}$ (using $D=780 \mathrm{kpc}$ ). The kinematical analysis of M31 is pursued here and new deep $21 \mathrm{~cm}$ observations obtained at the Dominion Radio Astrophysical Observatory (DRAO) are presented. A tiltedring model is fitted to a new HI velocity field, allowing the derivation of the position angle P.A. and inclination $i$ as a function of radius. We concentrate on the approaching half of the disk in order to compare our new results with those from C06. It is shown that the disk warping of M31 does not severely contaminate the kinematics of the neutral gas. As a consequence, the RC from $\mathrm{C} 06$ is in very good agreement with the new derived RC.
\end{abstract}

\section{Observations, results and future work}

HI-21cm data were obtained using the DRAO interferometer, combined with the DRAO $26 \mathrm{~m}$ single dish. Gaussian profiles were fitted to the Hi data-cube to extract the different moment maps. Figures $1 \mathrm{a}$ and $\mathrm{b}$ present the provisional integrated intensity map of the $21 \mathrm{~cm}$ emission line and its corresponding velocity field. Because M31 is known
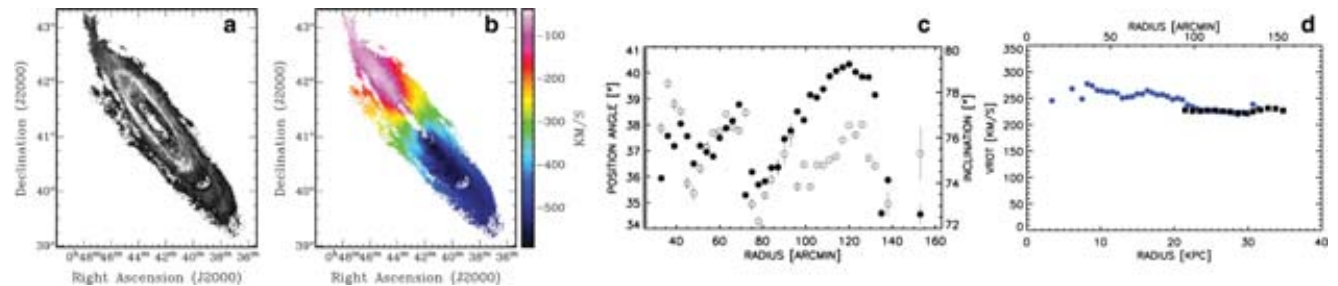

Figure 1. a-b HI integrated intensity and velocity field of M31 from new DRAO observations. c Radial profiles of P.A. and $i$ (full and empty dots resp.). d New Hi rotation curve of M31 (circles) and comparison with recent single dish velocities (C06, squares).

to have a warped disk, a tilted ring model has to be fitted to the velocity field. It allows to derive accurate kinematical parameters and rotation velocities as a function of radius. We focus on the approaching half of the disk in order to compare with the RC from C06. Figure 1c shows that $i$ does not vary significantly as a function of radius whereas the P.A. variation is more important. In other words, the warp of M31 is not severe and does not contaminate so much the Hi disk kinematics at large radius, at least in its approaching half. As a consequence, the RC from C06 is very similar to our new measurement (Fig 1d). Future work will consist of comparing the receding and approaching sides' kinematics in order to derive a global RC and a mass model for M31. 